\title{
Phänomenologisch-hermeneutische Struktur- und Prozessdiagnostik in der Existenzanalyse
}

\author{
Astrid Görtz
}

Online publiziert: 20. Mai 2020

(C) Der/die Autor(en) 2020

\begin{abstract}
Zusammenfassung Die neuere Existenzanalyse nach Alfried Längle ist durch die Entwicklung theoretischer Modelle auf Basis der existenzanalytischen Anthropologie gekennzeichnet, nämlich des Strukturmodells der existentiellen Grundmotivationen und des Prozessmodells der Personalen Existenzanalyse. Beide Theoreme stellen eine Grundlage für die Diagnose von personalen Ressourcen und Defiziten der Patient_innen am Beginn sowie im Laufe des Therapie-Prozesses dar. Entsprechend der phänomenologischen Grundhaltung der Existenzanalyse werden jegliche Erkenntnisse und Diagnosen während des therapeutischen Prozesses im Sinne einer phänomenologischen Epoché laufend überprüft und weiterentwickelt. Im vorliegenden Beitrag soll anhand einer Einzelfall-Analyse veranschaulicht werden, wie eine sowohl struktur- als auch prozessorientierte existenzanalytische Diagnostik den therapeutischen Prozess begleitet und nicht zuletzt eine systematische Erforschung der therapeutischen Wirkelemente im Wege qualitativer Einzelfallforschung ermöglicht.
\end{abstract}

Schlüsselwörter Existenzanalytische Diagnostik . Phänomenologisch-hermeneutische Diagnostik · Qualitative Einzelfall-Forschung

\section{Phenomenological-hermeneutic diagnosis of} structure and process in Existential Analysis

Summary The newer Existential Analysis of Alfried Längle is characterized by the development of theoretical models on the basis of existential analytical anthropology, particularly by the structural model of existential fundamental motivations and the process

\section{A. Görtz ( $\square)$}

Breitenfurter Straße 376/10/17, 1230 Wien, Österreich astrid.goertz@existenzanalyse.org model of Personal Existential Analysis. Both theorems constitute a basis for the diagnosis of personal resources and deficits of the patient in the beginning as well as during the process of therapy. In line with phenomenological Epoché, all insights and diagnoses are continuously reviewed and evolved in the course of therapy, in accordance with a phenomenological attitude which is fundamental to Existential Analysis. By means of an individual case study, the contribution on hand is thought to demonstrate how structure-oriented as well as process-oriented existential analytical diagnostics support the process of therapy, last but not least enabling systematic research on effective therapeutic elements through qualitative individual case research.

Keywords Existential analytical diagnostics . Phenomenological-hermeneutic diagnostics . Qualitative individual case research

\section{Diagnostizieren in der Existenzanalyse}

Der Ansatzpunkt der Existenzanalyse ist das „PersonSein in der Welt“, worin das „vor die Wahl gestellt Sein“ des Menschen zentrale Aufgabe ist (Längle 2005). Als Ziel sieht die Existenzanalyse, dass der Mensch von seinen Wahlmöglichkeiten Gebrauch machen kann, mit dem Ziel, zu der Haltung eines „Lebens mit innerer Zustimmung“ (d.h. in Freiheit und Verantwortung) zu gelangen. In der Regel geht es vor allem um das Finden eines Zugangs zur eigenen Emotionalität, welche die Basis einer authentischen Lebensführung darstellt (Längle 2005).

Der Sinn der Diagnostik ist nach Längle die Einschätzung eines störenden oder leidvollen Phänomens (eines „Problems“) hinsichtlich seiner Behandlungsbedürftigkeit, der Störungsursache und -Zusammenhänge, der Prognose (Therapieerwartung, Hin- 
dernisse und Gefahren während der Therapie) und schließlich eine Abstimmung der Erkenntnisse mit den Methoden einer optimalen Behandlung, inklusive der ökonomischen Kommunikation mit Fachleuten.

Die existenzanalytische Diagnostik geht als phänomenologische Diagnostik aus von dem, was den Patienten bewegt, und richtet ihre Aufmerksamkeit auf die existentiellen Fähigkeiten und Erfordernisse des Patienten. Diese werden durch die Klärung der Voraussetzungen für ganzheitliche Existenz (Grundmotivationen) und der Begegnungsfähigkeit mit sich und der Welt (Personale Existenzanalyse) erhoben. (Längle 2005, S. 85)

\section{Exkurs: Paradigmen der Psychotherapie- Forschung}

Diagnostik kann als Instrument der Forschung betrachtet werden. Mit unterschiedlichen ForschungsParadigmen gehen verschiedene diagnostische Strategien einher. Besondere Bedeutung erhalten diese vor allem für die Wirksamkeitsforschung.

Folgen wir dem italienischen Schriftsteller, Philosophen und Professor für Semiotik Umberto Eco (1932-2016), so gibt es zwei Kriterien für wissenschaftliches Arbeiten: erstens ein systematisches Vorgehen bei der Erkenntnisgewinnung und zweitens eine intersubjektive Nachprüfbarkeit. Beide Kriterien sind erfüllt, wenn Forscher_innen entweder schon vorhandenes Wissen überprüfen - wie Forschung klassisch in den Naturwissenschaften bzw. in der quantitativen Forschung betrieben wird. Dies geschieht im Kontext der Beweissicherung. Wissenschaftliches Vorgehen ist aber ebenso im Kontext der Entdeckung möglich, wie dies in den Geisteswissenschaften bzw. im Rahmen der qualitativen Forschung üblich ist. Letzterer erlaubt und erfordert einen wesentlich breiteren Zugang an Forschungsmethoden (Eco 1993).

Der Psychotherapie-Forscher Gottfried Fischer (1944-2013) knüpft an Umberto Eco an, wenn er für die Psychotherapie-Wissenschaft einen kreativen Zugang fordert, der sich von den Vorgaben der angrenzenden Wissenschaften, nämlich von der biologischen Psychiatrie einerseits und der experimentellen Psychologie andererseits, befreit. Fischer schlägt eine Kombination aus den beiden genannten Ansätzen vor, und zwar in Analogie zur Aufklärungsarbeit einer Detektiv_in, die_der einen Kriminalfall lösen soll. Diese_Dieser zieht einerseits Schlussfolgerungen aus Beobachtungen - dies bezeichnet man als induktives Vorgehen oder bottom up-Vorgehen. Und andererseits wendet sie_er vorhandene - in diesem Fall kriminologische - Theorien an, in einem top downVorgehen oder auch deduktiven Vorgehen. In der Praxis muss die Detektiv_in - und in Analogie dazu die Psychotherapie-Forscher_in - beide Vorgehensweisen kombinieren, um eine bisher vielleicht einzigartige Konstellation von Ereignissen aufzuklären. Dieses
Vorgehen bezeichnet Fischer als Abduktion oder „Ableitung“. Sie spiegelt den methodischen Zugang der Einzelfallforschung wider und entspricht auch dem psychotherapeutischen Vorgehen selbst, denn letztlich entwickelt ja die Therapeut_in für jede einzelne Patient_in das therapeutische Vorgehen auf Basis ihres_seines theoretischen und klinischen Vorwissens in Abstimmung mit der individuellen Problemlage und den Ressourcen der Patient_in jeweils neu (Fischer 2011).

Für die Suche nach Wirkelementen im psychotherapeutischen Prozess kann folgende Parallele gezogen werden: Das deduktive Vorgehen hat eine Entsprechung in den quantitativ-nachprüfenden Methoden der empirischen Psychologie bzw. der biologischen Psychiatrie, wobei von beobachtbaren Daten und Fakten, die als „Operationalisierungen“ von „Variablen“ auf Basis einer vorhandenen Theorie verstanden werden, mittels numerisch-statistischer Algorithmen auf die Theorie rückgeschlossen wird (im Sinne von „deducere“, lat. = rück-führen).

Das induktive Vorgehen hat eine Entsprechung in den qualitativen Methoden, die in der PsychotherapieProzess-Forschung schon seit den 1950er-Jahren eine Rolle spielen und in den letzten Jahren an Bedeutung gewonnen haben (Braakmann 2015).

\section{Der diagnostische Prozess in Psychologie und Psychiatrie}

Innerhalb des psychologischen Diagnostizierens wird streng deduktiv vorgegangen, in der Anwendung von quantitativen Fragebögen und Tests. Durch empirisch-statistisch untermauerte Algorithmen werden eindeutige Zuordnungen zwischen bestimmten Symptom-Ausprägungen und zugrunde liegenden Störungsbildern (z.B. einer Depression, einer Angststörung, etc.) getroffen. Natürlich können dabei immer nur Wahrscheinlichkeits-Aussagen getroffen werden - die psychologische Diagnostik beruht auf Inferenz-Statistik und ist immer messfehlerbehaftet.

Auch die psychiatrische Klassifikation nach dem ICD-10 oder dem DSM-IV erfolgt deduktiv, denn sie folgt mehr oder weniger eindeutigen Zuordnungs-Regeln. Klassifikationen beruhen auf einer Deskription der Symptome durch die Patient_innen und mit Hilfe von Diagnose-Checklisten, nach welchen die explorierende Psychiater_in vorgeht. Dabei wird nicht der Anspruch erhoben, das Leiden der Patient_innen auf einer subjektiven Ebene zu verstehen. Unklare Schilderungen, Erinnerungsfehler, reduzierte Ausdrucksmöglichkeiten sowie die bekannten Effekte der Simulation und Dissimulation bzw. Aggravation auf Seiten der Patient_in können die Aussagekraft der Symptomschilderung reduzieren. Eine im Rahmen der klinischen Routine unter Zeitdruck getroffene Klassifizierung kann die Genauigkeit der Zuordnung reduzieren. Dies alles kann in der Folge zu Fehldiagnosen führen. 
Abb. 1 Personale Existenzanalyse (PEA) nach Längle (1999)
PEA 2

Was halte ich grundsätzlich davon?

(Stellungnahme)

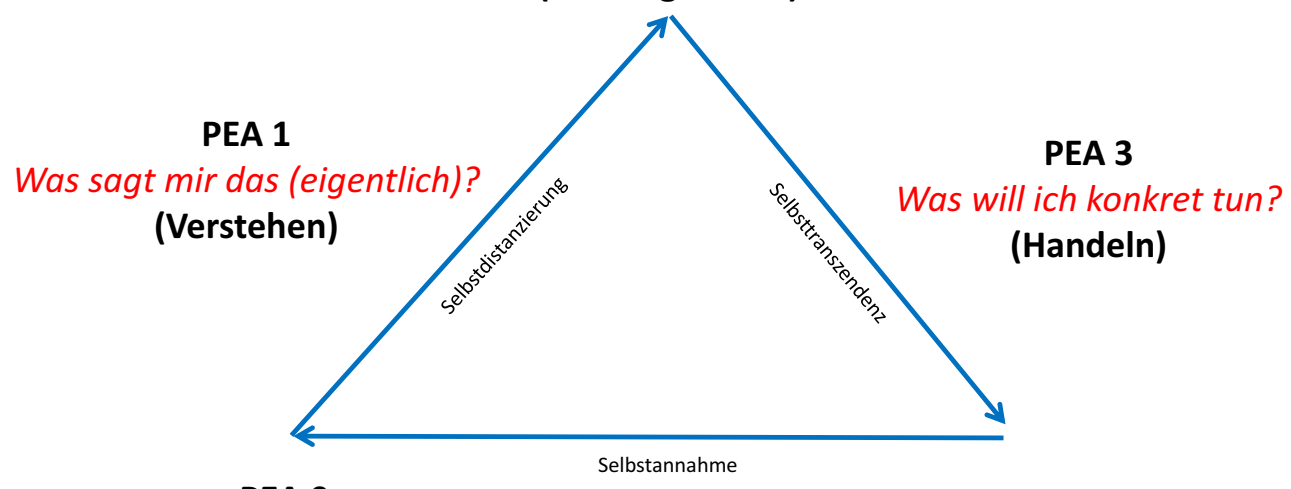

PEA 0

Was liegt vor?

(Daten, Fakten)

\section{Phänomenologisch-hermeneutisches Forschen in der Existenzanalyse}

Induktives Vorgehen entspricht einem phänomenologischen Prozess, wie ihn Alfried Längle in den Schritten der Personalen Existenzanalyse für Therapie und Beratung beschrieben hat. Die Personale Existenzanalyse (kurz: PEA) ist eine Methode zur Mobilisierung personaler Kräfte, welchen im existenzanalytischen Psychotherapieprozess zum Durchbruch verholfen werden soll (siehe Abb. 1). Unter „Person“ wird in der Tradition der Existenzphilosophie das Geistige, das Freie und sich Verantwortende im Menschen verstanden (Frankl 1959, zit. n. Längle 1999). Als Charakteristika des Person-Seins werden drei spezifische Eigenschaften in den Vordergrund gestellt.

Als Person ist der Mensch ansprechbar. ... Er ist ein Sprechender, weil ,es in ihm spricht. Als Person kann der Mensch verstehen, kann die Zusammenhänge und Beweggründe von anderen Menschen und von sich selbst finden, sich darin einfinden, sich daraus verstehen und darauf Bezug nehmen. ... Der Mensch ist ein Antwortender, weil er als ein im Wesen Sprechender nicht bei sich bleiben will, sondern auf Kommunikation, Austausch, Begegnung und Andersheit angelegt ist. ... Analog zu diesen drei, Begegnungscharakteristika' der Person finden sich innere Vorgänge. Im subjektiven Erleben empfängt der Mensch dort, wo er angesprochen ist, einen Eindruck, führt ihn das Verstehen zu persönlichen Stellungnahmen und erlebt er sich im Antworten als sein Inneres zum Ausdruck bringend. (Längle 1999, S. 21; Hervorhebungen im Original)

Die Schritte der Personalen Existenzanalyse im Einzelnen sind:
- Deskription der Daten und Fakten: Was liegt vor?

- Eindruck, phänomenologische Wesensschau: Welche Gefühle macht das? Welche Bewegung stellt sich spontan ein? Was sagt mir das (eigentlich)?

- Personale Stellungnahme: Verstehe ich mich? Was bewegt mich dabei? Verstehe ich den anderen? Was verstehe ich nicht? Was spüre ich im tiefsten Innersten dazu? Was halte ich grundsätzlich davon? Was sage ich persönlich dazu? Was würde ich da am liebsten und im Grunde tun wollen?

- Ausdruck, Handeln: Was davon will ich konkret tun? Wieviel davon mag ich preisgeben? Wem? Passt es bei diesem Menschen? Wie und mit welchen Mitteln mache ich es am besten? Bei welcher Gelegenheit?

Wenn Forschen als Erkenntnisprozess verstanden und Einzelfall-Diagnostik als Prozess des Verstehens von Person(en) betrachtet wird (siehe Abb. 2), kann die Personale Existenzanalyse diesen Prozess anschaulich beschreiben: Ausgehend von Daten und Fakten (PEA 0: Deskription) entwickeln die Forscher_in bzw. Therapeut_in (am besten in Personalunion) und die Patient_in im Weg des Dialogs über den jeweils subjektiven Eindruck und über die phänomenologische Verdichtung desselben auf das Wesentliche hin (PEA 1: phänomenologische Wesensschau) ein gemeinsames, intersubjektives Verständnis, das als diagnostische Aussage aufgefasst werden kann (in Analogie zu PEA 2: personale Stellungnahme - „so verstehen wir die Person/das Anliegen/Problem“). Der letzte Schritt des induktiven Vorgehens wird im Kontext des Forschens als (Weiter-)Entwickeln anthropologischer Theoreme und Konzepte verstanden (in Analogie zu PEA 3: Handeln - „so kann es verallgemeinert werden“). So kann etwa die Erfindung und Entwicklung der existentiellen Grundmotivationen oder auch der Personalen Existenzanalyse selbst als Erkenntnisprozess verstanden werden. 
Abb. 2 Forschen als Erkenntnisprozess

\author{
Problem-Diagnose \\ (kommunizierbar zw. Therapeut/Patient) \\ Wie verstehen wir die Person/das Anliegen/das Problem? \\ PEA 2
}

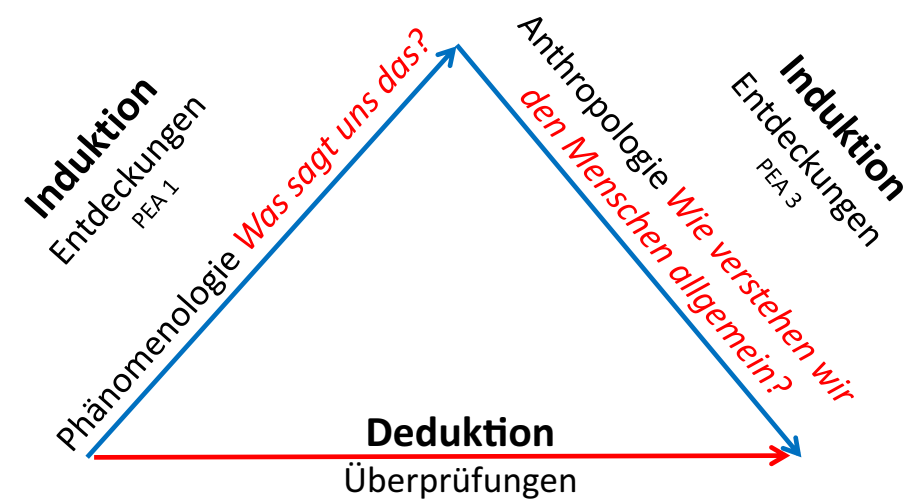

Beobachtungen, Daten, (Operationalisierungen, Fakten Was liegt vor? statist. Algorithmen)

verallgemeinerte wiss. Aussage (Theorie bzw. Anthropologie) intersubjektiv kommunizierbar in der Scientific Community

\section{Der diagnostische Prozess in der Existenzanalyse}

In der Existenzanalyse erfolgt der diagnostische Prozess anfangs induktiv - im Verstehen des subjektiv empfundenen Leidens der Patient_in - und in weiterer Folge abduktiv.

Neben den subjektiven Erwartungen, die der Patient in die Therapie hat, geht es auch um die fachliche Feststellung von Störelementen und krankheitsbedingten Ursachen. Das verlangt einerseits eine phänomenologische, einfühlsame Vorgehensweise und andererseits für die Erklärungen des Krankheitsbildes die Kenntnis der speziellen, existenzanalytischen Psychopathologie auf der Basis von anamnestischen Rückfragen. ... Für das Finden der personalen Ressourcen dient als schematischer Hintergrund das Strukturmodell der Existenzanalyse, die personalen Grundmotivationen. ... Zusätzlich werden aber auch die Fähigkeiten hinsichtlich der personalen Dynamik auf dem Hintergrund der Personalen Existenzanalyse eingeschätzt. (Längle 2005, S. 88f.)

Nach dem Prozessmodell der Personalen Existenzanalyse erfolgt zunächst eine möglichst genaue Exploration der Umstände des Leidens (PEA 0: Deskription der Daten und Fakten; Was liegt vor?). Für die Klarheit der Deskription wird ausschlaggebend sein, wie gut die Patient_in ihr Leiden wahrnehmen und beschreiben kann. Die diagnostizierende Therapeut_in ist gefordert, so detailliert wie möglich nachzufragen, ohne dabei etwaige Schutzmuster der Patient_in zu durchbrechen. Zum Beispiel könnten sehr lückenhafte Schilderungen Hinweise auf eine reduzierte Wahrnehmungsfähigkeit auf Grund traumatischer Erfah- rungen sein. Alles, was die Patient_in von sich zeigt und sagt - auch das, was sie nicht zeigen/sagen kann -, ist bedeutsam und wird in einer offenen phänomenologischen Haltung entgegengenommen.

Als nächstes folgt der Schritt des phänomenologischen Verdichtens, welches noch stärker die Subjektivität der Therapeut_in einbezieht (PEA 1: Wie wirkt die Patient_in in ihrem_seinem Leiden auf mich? Wie fühlt es sich bei mir an? Was sagt mir das?). Über das emotionale Mitschwingen der Diagnostiker_in/ Therapeut_in mit dem Leiden der Patient_in, in der Haltung einer nach innen gerichteten Aufmerksamkeit wird die emotionale Wirkung erfühlt und der wesentliche Kern dessen, worunter die Patient_in leidet, worum es ihr_ihm in ihrem_seinem Leiden geht, was ihr_ihm fehlt, erfasst. Die therapeutische Wirkung ist implizit im diagnostischen Prozess enthalten, wenn die Patient_in sich in diesem Mitschwingen der Therapeut_in verstanden fühlt.

Schließlich gipfelt der diagnostische Prozess in einer gemeinsam mit der Patient_in gefundenen Stellungnahme (PEA 2): So steht die Patient_in zu ihrem_seinen aktuellen Problem, so kann sie_er es benennen. Eine erste Orientierung ist gefunden, der Ausgangspunkt der Therapie ist markiert.

Nun folgt noch ein letzter Schritt, nämlich die $B e$ darfsanalyse: Was braucht sie_er? Sie bezieht sich auf die Lebensumstände der Patient_in und entspricht im Modell der PEA dem Handeln (PEA 3).

Eng mit dem letzten Diagnoseschritt verbunden ist die Bedarfsanalyse. Es geht darum, wie der Therapeut auf der Grundlage seines Fachwissens und seiner Beobachtungen die existentielle Situation des Patienten/Klienten hinsichtlich dessen 
einschätzt, was dieser unmittelbar zur Verbesserung seiner Lebenssituation benötigt. Diese fachliche Einschätzung verlangt vom Therapeuten den Einsatz derselben Fähigkeiten der Personalen Existenzanalyse. (Längle 2005, S. 90)

An dieser Stelle sei gesagt, dass der Prozess der Personalen Existenzanalyse keinem starren Ablauf folgt. Sobald sich Lücken auftun, kehrt die Diagnostiker_in/ Therapeut_in zum vorherigen Schritt zurück bzw. wird der Prozess immer wieder neuerlich durchlaufen. Auf diese Weise wird das Wahrnehmen, Verstehen und Stellungnehmen auf dialogischem Wege immer weiter konkretisiert, vertieft, geklärt bzw. an den äußeren Lebensumständen (imaginativ oder real) handelnd überprüft und abgesichert.

\section{Hermeneutisches Vorgehen: Herstellen eines Bezugs zur Psychopathologie}

Der oben beschriebene letzte Schritt stellt den Übergang von einer phänomenologischen, an den Ressourcen der Person orientierten Diagnostik zum therapeutischen Handeln dar.

Eine andere Möglichkeit des Vorgehens ist es, an dieser Stelle die phänomenologische Haltung im engeren Sinn zu verlassen und nach den Regeln der Hermeneutik vorzugehen, indem auf vorhandene Theorien Bezug genommen wird. In dieser Sichtweise wird das therapeutische Handeln klar vom diagnostischen Handeln unterschieden. In diagnostisch-hermeneutischer Betrachtung kann der letzte Schritt (PEA 3) im Sinne eines Erkenntnisprozesses als ein innerer Handlungsschritt der diagnostizierenden Therapeut_in definiert werden, der auf die Herstellung eines Bezugs zu vorhandenen psychopathologischen Theorien abzielt. Dieser „innere“ Schritt ist zwar von der Haltung her dialogisch, geschieht aber nicht in Absprache mit der Patient_in. Er dient vielmehr der theoretischen Orientierung der Therapeut_in als Fachexpert_in. Die Therapeut_in ist an dieser Stelle zugleich Forscher_in, die im Dialog mit ihren Fachkolleg_innen und mit der gesamten Fachliteratur steht.

Der induktive Prozess wird hier abgelöst durch ein abduktives (ableitendes) Vorgehen, unter Einbeziehung psychopathologischer Theorien, zum Beispiel Konzepte von Diagnosen, klinischen Bildern, Mustern von Coping-Reaktionen, etc. - alle diese Theorien basieren letztlich auf dem Strukturmodell der Existenz, den vier existentiellen Grundmotivationen nach Alfried Längle. In aller Kürze seien sie hier umrissen (Näheres bei Längle 2013):

1. Grundmotivation: Sein-Können; Voraussetzungen dafür sind die Erfahrung von Schutz, Raum und Halt; Themen: Grundvertrauen, Seinsgrund; Psychopathologie: Grundangst

2. Grundmotivation: Leben-Mögen; Voraussetzungen dafür sind die Erfahrung von Nähe, Zeit und Bezie- hung; Themen: Grundwert, Grundbeziehung zum Leben; Psychopathologie: Depression

3. Grundmotivation: So-Sein-Dürfen; Voraussetzungen dafür sind die Erfahrung von Gerechtigkeit, Beachtung und Wertschätzung; Themen: Selbstwert, Authentizität, Ethik; Psychopathologie: Hysterie, Narzissmus, Borderline

4. Grundmotivation: Sinnvolles-Wollen; Voraussetzungen dafür sind ein Tätigkeitsfeld, ein Strukturzusammenhang und ein Wert in der Zukunft; Themen: Existenz, Erfüllung; Psychopathologie: existentielle Leere, Para-Existentialität, Suizidalität

Die Anwendung von theoretischen Konzepten auf den Einzelfall bezeichne ich als „klinische Methode“. Dabei handelt es sich um ein In-Beziehung-Bringen von individuellen Phänomenen mit aus der Theorie abgeleiteten Hypothesen - ganz analog zu der von Gottfried Fischer beschriebenen „Detektiv-Methode“. Kein Einzelfall wird zur Gänze in eine vorhandene Theorie passen, daher müssen Diagnosen immer phänomenologisch offen bleiben. Weil die vorhandenen Kategorien nie alle Fälle erklären können, werden andererseits über Fall-Vergleiche neue diagnostische Kategorien bzw. Typologien kreiert und auf diese Weise vorhandene Theorien weiterentwickelt.

Ein einfaches Beispiel zur Differenzierung einer diagnostischen Kategorie ist der fiktive Vergleich von Patient_innen, die an einer Angststörung leiden. Durch Fall-Vergleiche lässt sich leicht zeigen, dass sich Patient_innen mit einer „Erwartungsangst“ deutlich von solchen unterscheiden, die an einer „Grundangst“ leiden - obwohl vielleicht in einer ersten (deskriptiven) Diagnosestellung für beide der Begriff „Angststörung“ zutrifft. Für eine weiter gehende Unterscheidung kann (abduktiv) auf die Theorie der existentiellen Grundmotivationen zurückgegriffen werden. So zeigt sich bald, dass es sich um deutlich unterscheidbare Störungsbilder handelt, die auch unterschiedliche therapeutische Strategien erfordern. So wäre einer Patient_in mit einer Grundangst-die aus einem Defizit im Bereich des Grundvertrauens (erste Grundmotivation) entsteht - mit einem stützenden, haltgebenden Beziehungsangebot in der Therapie zu begegnen, während eine Patient_in, die an einer Erwartungsangst leidet - die einem Defizit in der dritten Grundmotivation entspringt, also letztlich einem mangelnden GesehenWerden als Person - zur Überwindung ihrer_seiner Angst die personale Stellungnahmen herausfordernde Angst-Konfrontation braucht. Insgesamt spiegelt diese Vorgangsweise die Arbeit der „forschenden Praktiker_in“ wider, die ihr klinisches Wissen durch Erfahrung ständig weiterentwickelt.

Für das therapeutische Vorgehen hat die Diagnose auf Basis der Grundmotivationen also wichtige Konsequenzen, vor allem was die Gestaltung der therapeutischen Beziehung betrifft. Die enge Verknüpfung zwischen phänomenologisch-hermeneutischer Diagnostik und den Wirkelementen des Therapie-Prozes- 
Abb. 3 Zwei Richtungen des diagnostischen Prozesses

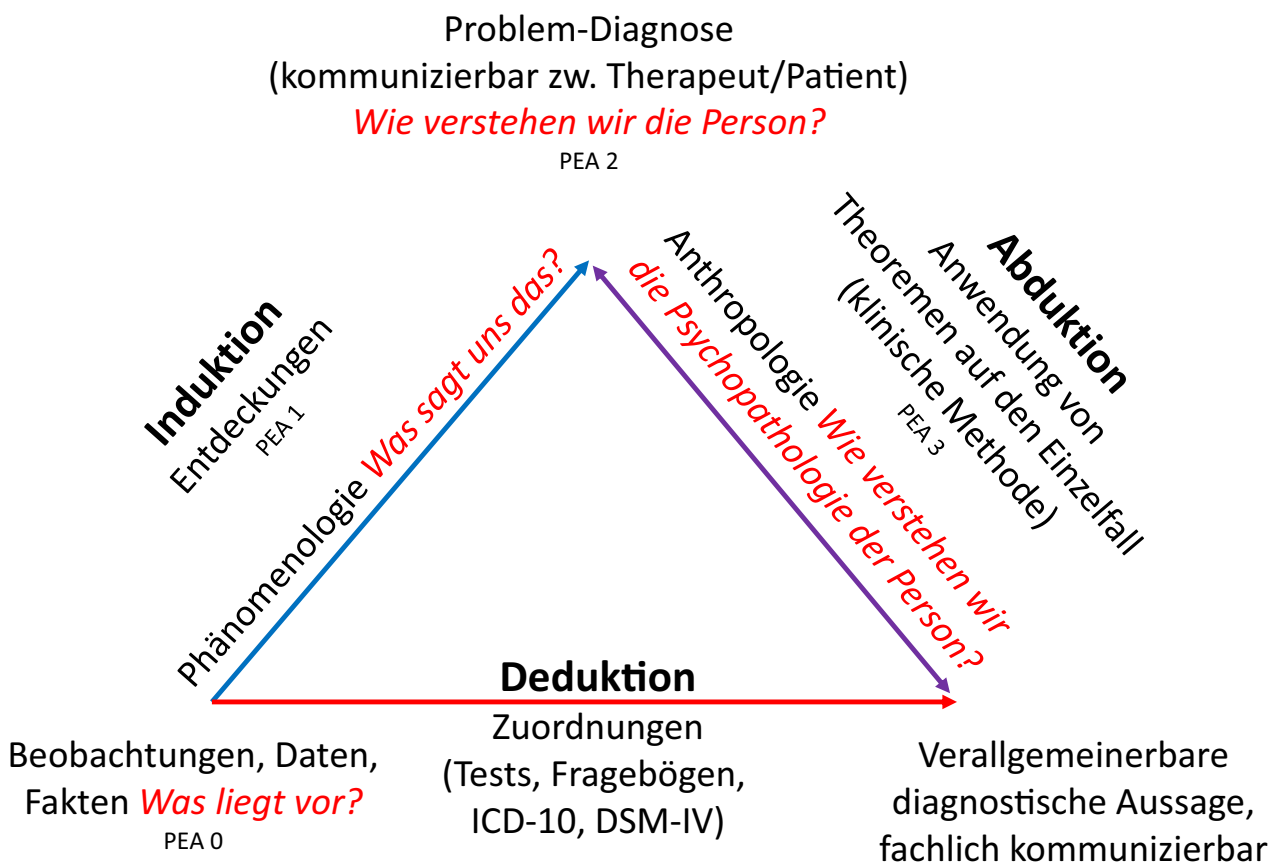

ses soll im Folgenden an Hand einer Einzelfall-Studie veranschaulicht werden.

\section{Empirische Untersuchung: Therapierückblick mit eigenen Patientinnen}

In einer Einzelfall-Studie wurden Wirkelemente von existenzanalytischen Psychotherapien herausgearbeitet (Görtz 2019). Dazu wurden Interview-Ausschnitte aus rückblickenden Gesprächen mit eigenen Patientinnen analysiert, und zwar in Hinblick auf die Frage „Was hat geholfen?". Im Sinne einer Triangulation der Forschungsmethoden wurden zusätzlich Selbsteinschätzungs-Fragebögen zur Symptombelastung (SCL90-S, Franke 2014) und zu personal-existentiellen Ressourcen (Existenzskala nach Längle et al. 2000) vorgegeben.

Die Auswahl der Interview-Themen orientierte sich am so genannten Change Interview nach Elliott (1999), zuvor wurde ein freier Rückblick mit spontan gestellten Fragen zum Therapieprozess durchgeführt.

Die Auswertung folgte den Prinzipien der Induktion sowie der Abduktion, wie von Fischer (2011) beschrieben bzw. parallel zu den Schritten der Personalen Existenzanalyse (PEA), wie in Abb. 3 dargestellt.

Die ursprüngliche Studie beinhaltet drei EinzelfallAnalysen, von denen ein Fall herausgegriffen wird (vgl. Görtz 2019).

\section{Patientin Elisabeth (34 Jahre)}

\section{Diagnostik nach ICD-10}

Die klinische Diagnose lautete „generalisierte Angststörung“. Elisabeth litt seit Monaten unter starken Angstsymptomen mit körperlicher Anspannung und
Schwindelgefühlen, die sich zu richtigen Angstanfällen steigern konnten. Sie ging nicht mehr aus dem Haus und konnte nicht allein sein. Der Mann, ein selbstständiger Ziviltechniker, richtete seinen ganzen Tagesablauf nach seiner Frau. Um die damals sechsjährige Tochter kümmerte sich ebenfalls der Vater. Elisabeth war über einige Wochen stationär psychiatrisch behandelt worden, bevor sie zu mir in die Praxis kam.

Wichtig ist zu wissen, dass Elisabeth vor ca. zehn Jahren auf Grund einer Entzündung des Sehnervs die Diagnose „Multiple Sklerose“ gestellt bekam. Elisabeth hatte seither keinen weiteren Schub.

$\mathrm{Zu}$ Therapiebeginn und nach neun Monaten ersuchte ich Elisabeth, einige Selbsteinschätzungs-Fragebögen auszufüllen (siehe Abb. 4). ${ }^{1}$ Die Testergebnisse zeigen eine deutliche Reduktion von Elisabeths Symptomen, vor allem in den Bereichen Ängstlichkeit, phobische Angst, Somatisierung und Zwanghaftigkeit. Im Bereich der personal-existentiellen Fähigkeiten zeigte sich eine Zunahme vor allem im Bereich der Selbsttranszendenz.

Das rückblickende Interview fand neun Monate nach Therapiebeginn statt. Die Therapie ging über ein Jahr weiter.

\section{Therapiewirkungen aus Patientinnen-Sicht}

Die im ersten Schritt einer Deskription der Inhalte/ Fakten (analog PEA 0) gefundenen Aussagen der Patientin werden im Folgenden aufgelistet und gleich

\footnotetext{
${ }^{1}$ Die Testungen wurden im Rahmen eines Forschungsprojekts der Gesellschaft für Logotherapie und Existenzanalyse durchgeführt. Sie erfolgten nach Information und mit dem Einverständ-
} nis der Patientin. 


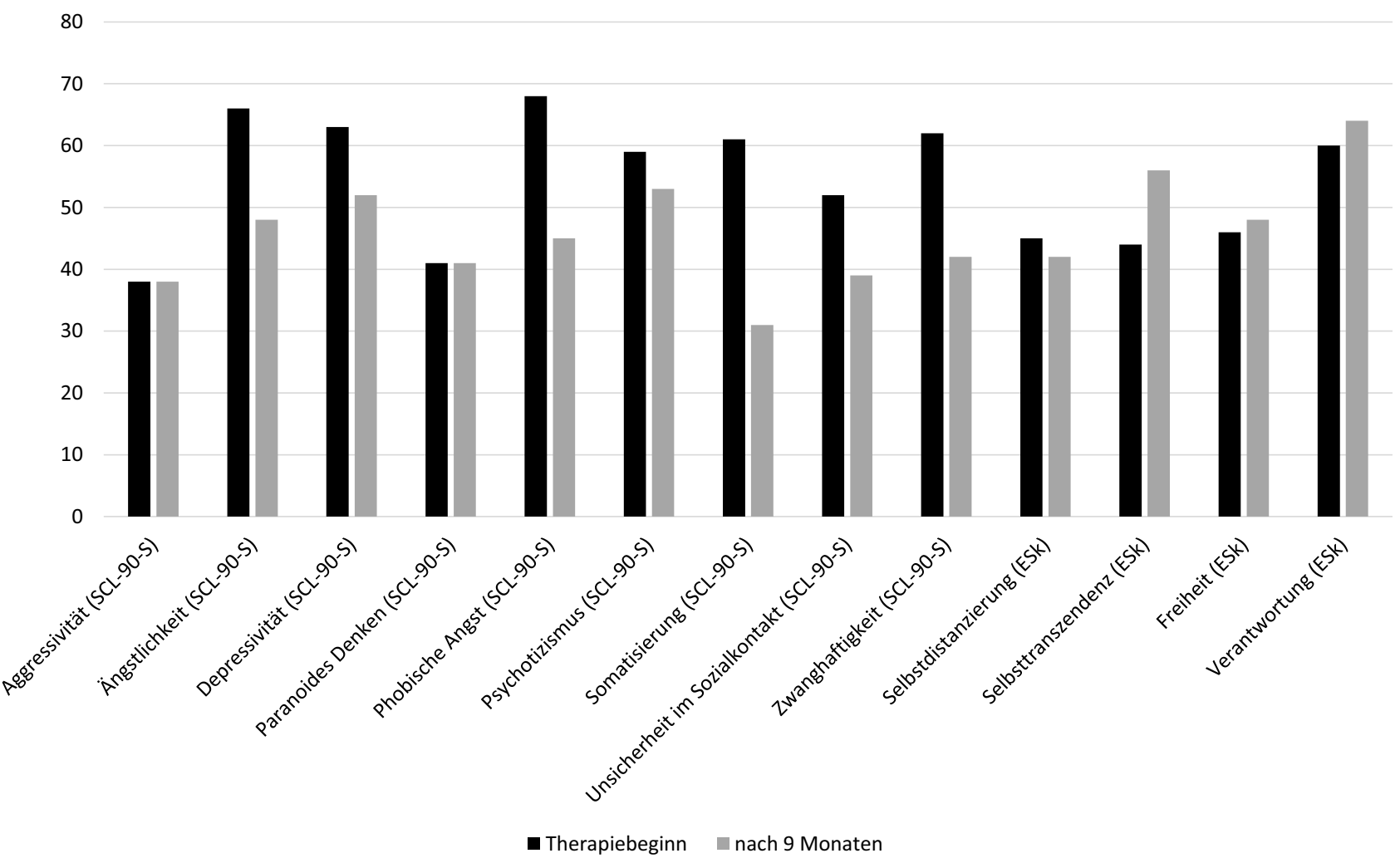

Abb. 4 Selbsteinschätzungsmaße Patientin Elisabeth

zu Kategorien in Hinblick auf die Beantwortung der Fragestellung zusammengefasst. In Klammern werden beispielhaft Original-Aussagen angeführt.

Zur Fragestellung „Was hat geholfen“ nannte Elisabeth folgende Themen:

- Reden („das Reden hat mir immer gutgetan“)

- das Aushalten der Angst („Bis zu dem Zeitpunkt dann, wo ich das irgendwie offenbar kapiert habe, dass ich das annehme und aushalte. ... Das war der Knackpunkt. ... und durch dieses Gefühl, ... da haben Sie gesagt, wie, wie fühlt sich das an? Das Aushalten ... in den Beinen ist so, wie so eine Spannung. ... dann ... habe ich irgendwie kapiert, das hilft mir. Und dann habe ich mir das immer vorgesagt, dieses ,Ich kann', ,Ich nehme die Ängste an“ und kann die Symptome aushalten. Und das hat mir total geholfen.... auch ... der Herzschlag, plötzlich war ich beruhigt.")

- Bestärkung durch die Therapeutin („und da haben Sie mir immer gesagt [lacht] Sie halten das aus.")

- ein gedanklicher Mechanismus, der ihr zur Verfügung stand (,von da habe ich es dann kapiert.... [hab’ ich] wirklich einen Mechanismus gehabt, der mir geholfen hat.")

- die Ressourcen-Übung „inneres Team“, die sie mit einer anderen Imaginations-Übung aus der Therapie kombinierte

- Eine hilfreiche Aktivität, die sie etwa zwei Monate nach Therapiebeginn außerhalb der Therapie entdeckte und entwickelte, war die Ikonen-Malerei („Und da habe ich gewusst, ich brauche diese Vormittage, um das zu machen.")

Auf die Frage nach dem Verlauf der Veränderung kontinuierlich oder in Form von Wendepunkten - beschreibt sie eine weitere kontinuierliche Verbesserung im Zusammenhang mit alltäglichen Themen (Freundinnen, Beziehung, Arbeit) und dem Erkennen ihrer Muster. Letzteres ist ein entscheidendes Wirkelement. In der Reflexion entwickelte sich daraus eine Analyse, wie alles angefangen hat

\section{E: „Ich habe ja Angst gekriegt, wie ich heimgekom- men bin.... Ich habe mir gedacht, was tue ich $z u$ Hause, da bin ich wieder in dieser alten, meiner alten Umgebung, die mir ja auch die Angst ge- bracht hat. ... Da hat alles angefangen, zuhause. Und jetzt ... mit dem Mechanismus, ich nehme die Ängste an und ich kann das aushalten, ist es... besser geworden."}

Im Anschluss wurden die Fragen aus Elliotts Change Interview gestellt. Die Auswertung folgte denselben Schritten wie jene des freien Therapierückblicks. Dabei zeigt sich, dass die strukturierte Befragung bei Elisabeth die Wirkelemente noch prägnanter fassen kann. Die vorgegebene Struktur des Interviewleitfadens (teilweise mit Quantifizierungen) scheint Elisabeth Halt $\mathrm{zu}$ geben und entspricht ihrer eher ängstlichen Persönlichkeit. Im Change Interview wie- 
derholte Elisabeth die meisten der bereits analysierten Wirkelemente. Folgende Wirkung ist zentral:

I: Welche Veränderungen würden Sie an sich beschreiben, seit Beginn der Therapie?

\section{E: „Die Angst ... habe ich in den Griff bekommen."}

Außerdem nennt Elisabeth neue Themen. Auffallend sind die Klarheit und Präzision von Elisabeths Formulierungen. Sie benötigen keine weitere Zusammenfassung:

E: „Dann, diese Veränderung, zu sehen, wie andere ticken, das zu erkennen und damit zu arbeiten oder selbst zu überlegen, was spielt sich da ab. ... Das beinhaltet auch, dass ich, dass ich für mich Grenzen setzen kann, ja. Dass ich nicht alles reinlasse.... Ich bin ich jetzt sensibel oder hellhörig, ich weiß, ich muss bei Freundinnen aufpassen, und das ist schon ganz gut....

Dann, ich bin in gewissen Dingen ... lockerer geworden.

Dann habe ich auch bemerkt, dass man das Gehirn auch steuern kann, also dass das recht schnell geht, dass man eigentlich da so eine neue Bahn ziehen kann. Dann was für eine Kraft ich habe, die ich ja anders nutzen kann. Wenn ich die Kraft habe oder das Potenzial, mir selbst Krankheiten oder Taubheit in den, in den Beinen zuzufügen, wie könnte ich das positiv nutzen? ... Also dass ich mehr ins Positive gehe ... Dann, mit meiner Familie, dass ich da hoffentlich weiterhin offener reden kann. Dass ich mich traue, Dinge anzusprechen, wenn es, wenn es verlangt ist. [Ich sag mir oft:] ,So, versuche im Jetzt zu sein' und das intensiv wahrzunehmen, ob das jetzt Fleisch schneiden ist, kochen, was auch immer. Wenn etwas auf mich zukommt, zu schauen, wie, wie geht es mir damit einmal ... und nicht gleich irgendwie reagieren ins Außen, sondern genau versuchen zu überlegen, was spielt sich jetzt in mir ab oder wie geht es mir damit. ... ich finde, ich bin ganz anders, also es hat sich viel getan. ... [Ich bin] entspannter und aufmerksamer."

I: Wodurch, glauben Sie, sind diese Veränderungen zustande gekommen?

E: „Da waren einmal diese Mechanismen, die man anwenden kann, ob jetzt, dass man sich mit den Händen beschäftigt bis zu über, also bis diese Reise, ja, mit, oder dieses, diese Überlegungen mit diesen vier Figuren, die einem helfen, ja. [die Übung ,Inneres Ressourcen-Team', Anmerkung d. Verf.] Dann ... offen reden, also das waren einmal die Veränderungen, mit denen wir die kleinen Schritte bearbeitet haben. Dann dieser große Schritt, die Angst anzunehmen und auszuhalten, die Symptome - das war total der Knackpunkt.

Dann ... auch diese Beschäftigungen über den Tag ... dass ich einen Auftrag habe. Dann über das, etwas selbst gefunden ... das mit der Malerei, die mich dann beschäftigt hat. Dann über diese ... alltäglichen Dinge, die ich hier besprochen habe, wo ich dann erkannt habe ,Aja, so könnte das laufen" (lacht) oder so sind die Muster meiner Freundinnen, wie auch immer und ich verstehe sie."

I: Was war insgesamt hilfreich in der Therapie?

E: „Das Erkennen, dass diese kleinen Schritte helfen. Also diese Mechanismen, dass die helfen. ... wenn ich jetzt ... mit den Händen arbeite, ja, dann komme ich von dieser Gedankenschleife weg, also das zu erkennen, dass ich mir da auch helfen kann. Dann eben auch das anzunehmen und auszuhalten. Also, das, genau also das ist eigentlich entscheidend, dass man selber auch was dazu tun kann. (...)“

Phänomenologisch-hermeneutische Diagnostik: Wirkelemente aus dem Blickwinkel der Personalen Existenzanalyse (PEA) und der Grundmotivationen (GM)

Im Folgenden werden die auf induktivem Weg gewonnenen Kategorien den Theoremen der Existenzanalyse - den Grundmotivationen und der Personalen Existenzanalyse zugeordnet. Dabei wird die inhaltliche Ebene von der phänomenalen Ebene (bezogen auf das Wie und auf die Struktur von Elisabeths Aussagen) unterschieden.

\section{Inhaltliche Analyse der Antworten}

Auf einer inhaltlichen Ebene nennt Elisabeth bezüglich Veränderungen an sich selbst vor allem eine veränderte Wahrnehmung (sensibel, hellhörig, Schutz ihrer Grenzen im Sinn von nicht alles reinlassen, intensiv im Jetzt wahrnehmen, aufmerksamer, Erkennen von Mustern). Auch bezüglich der körperlichen Symptome zeigt sie eine veränderte Wahrnehmung im Sinne eines größeren Abstands zu ihren Ängsten (auch wenn sie dies selbst nicht so bezeichnet).

Auf die Frage nach dem Zustandekommen dieser Veränderungen beschreibt sie gedankliche wie reale Handlungen (Mechanismen, imaginierte Reise, Beschäftigungen) sowie das Reden (über ihre alltäglichen Schwierigkeiten). Der Knackpunkt war das Aushalten und Annehmen der Angst. Insgesamt hilfreich war es, sich selbst helfen zu können.

Die Therapie hat Elisabeth vorwiegend in den Bereichen Wahrnehmen (PEA 0) und Handeln (PEA 3) gestärkt. Die Schritte Verstehen (PEA 1) und personale Stellungnahme (PEA 2) bringt Elisabeth als Kompetenzen selbst mit.

Andererseits wird deutlich, dass es vorwiegend um eine Förderung in den Bereichen der ersten Grundmotivation ging. In der Betonung von Selbsthilfe sehen 
Abb. 5 Defizitäre Ausgangslage bei Elisabeth (Grundangst-Störung) aus Perspektive der Personalen Existenzanalyse

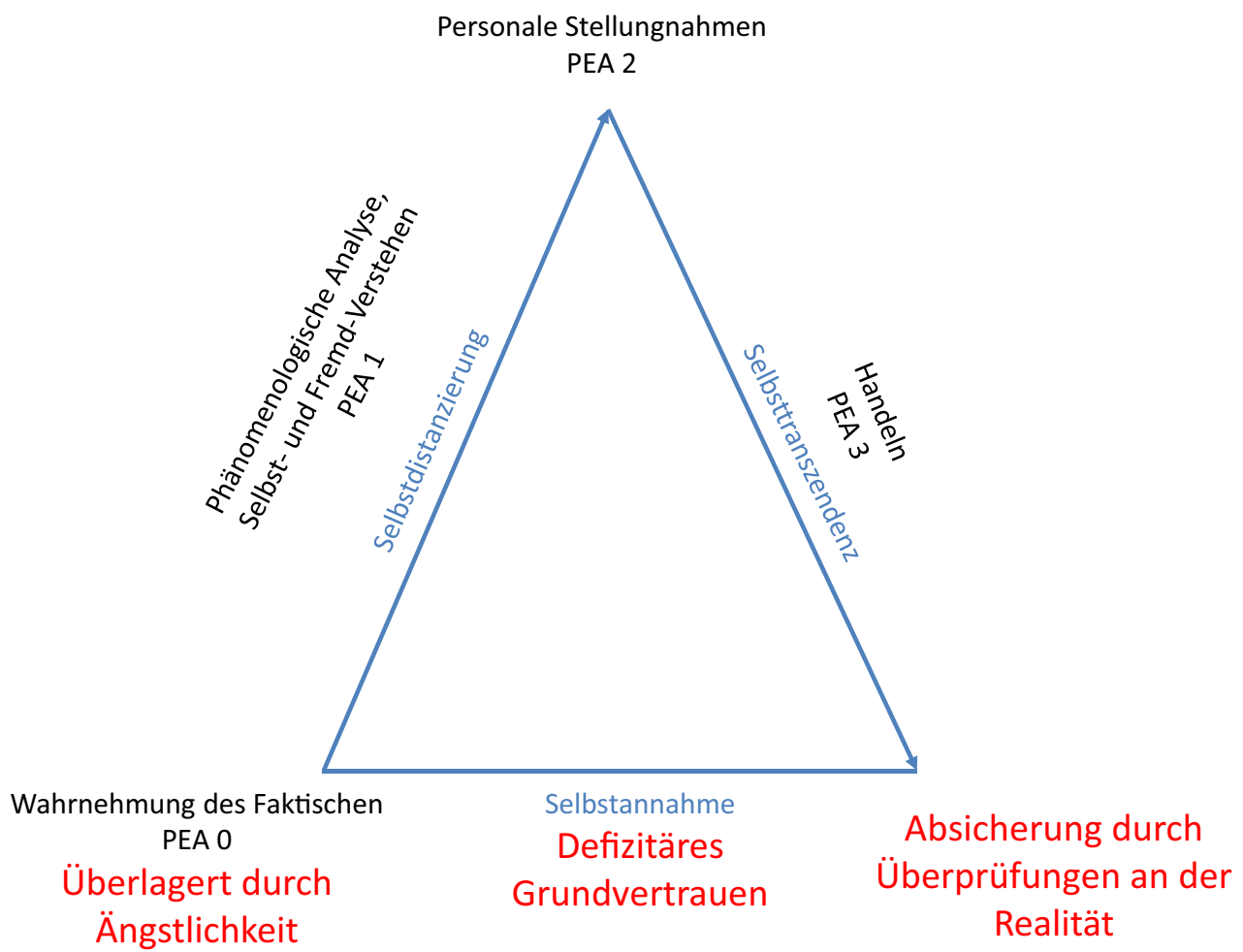

PEA 2 wir eine Stärkung der Fähigkeiten der dritten Grundmotivation als Ausdruck von Selbstständigkeit und Autonomie, allerdings wieder angebunden an das Sein-Können. Dieses Vorgehen entspricht den theoretischen Vorstellungen über die existenzanalytische Therapie von Ängsten, insbesondere im Kontext von Grundangst.

Der hier vorgestellte Abschnitt der Therapie mit Schwerpunkt in der ersten Grundmotivation hatte einerseits das Wahrnehmen im Hier und Jetzt und andererseits das Handeln im Fokus. Diese waren aus Perspektive der Personalen Existenzanalyse betrachtet die wichtigsten Wirkelemente.

\section{Elisabeths Antworten - phänomenologisch betrachtet}

Betrachten wir nun Elisabeths Sprechen als Ausdruck ihres Person-Seins im Sinne der phänomenologischen Haltung, die charakteristisch für die therapeutische Haltung ist, und stellen anschließend einen Bezug zu den Theoremen der Existenzanalyse her.

In der phänomenologischen Betrachtung erscheinen Elisabeths Antworten als nüchterne und klare, kognitiv betonte, emotional wenig angereicherte Stellungnahmen, so wie dies Elisabeths eher nüchterner rationalen Persönlichkeit entspricht. Emotionen zeigt Elisabeth öfters in einem Lachen, das von einer Gruppe von studentischen Auswertern als „nicht zum Inhalt passend“ und „unsicher, nach Bestätigung suchend" bezeichnet wurde. Auch Elisabeths Gesprächsstil, Worte oftmals zu wiederholen, macht den Eindruck von Unsicherheit. Elisabeth greift die Worte der Interviewerin immer wieder auf und bestätigt diese mit einem wiederholten zustimmenden „ja“. Es wirkt, als versuche sie, möglichst keine Fehler zu machen und „richtig“, im Sinne von vorgegebenen Erwartungen zu antworten. Dies mag der Grund sein, weshalb das strukturierte Interview Elisabeth besser entspricht als das freie Gespräch - die Struktur lässt weniger Spielraum für Fehler.

Wie lässt sich nun diese spezifische Umgangsweise mit ihren Themen aus dem Blickwinkel der Personalen Existenzanalyse verstehen? Elisabeth findet $\mathrm{zu}$ Stellungnahmen (PEA 2), jedoch sind diese sehr kognitiv gehalten. Das emotionale Mitschwingen (PEA 1) scheint für Elisabeth subjektiv kein Problem zu sein. Allerdings kann die etwas „leichtgewichtige“ Fundierung der Emotionalität als Risikofaktor zur Entstehung einer Grundangst-Störung betrachtet werden. Abb. 5 veranschaulicht die Schwäche in einer quasi „verkürzten“ Basis-Linie im Dreiecks-Modell der Personalen Existenzanalyse (in Analogie zu einer mangelhaften Selbstannahme auf Grund ihres defizitären Grundvertrauens). Daraus resultiert ein insgesamt „schmal abgestützter" Prozess im Sinn der Personalen Existenzanalyse, mit dem „Schwerpunkt“ in der Kognition. Sowohl das Verstehen (PEA 1) als auch die Stellungnahmen (PEA 2) gelingen zwar, jedoch ist das Wahrnehmen (PEA 0) durch Ängste eingeschränkt. Das Handeln (PEA 3) ist unfrei, da für Realitäts-Prüfungen im Sinne von Absicherungen funktionalisiert. Dies ergibt das klinische Bild einer Grundangst-Störung. 
Abb. 6 Verbreiterung der ontologischen Basis durch Therapie der ersten Grundmotivation bei Elisabeth

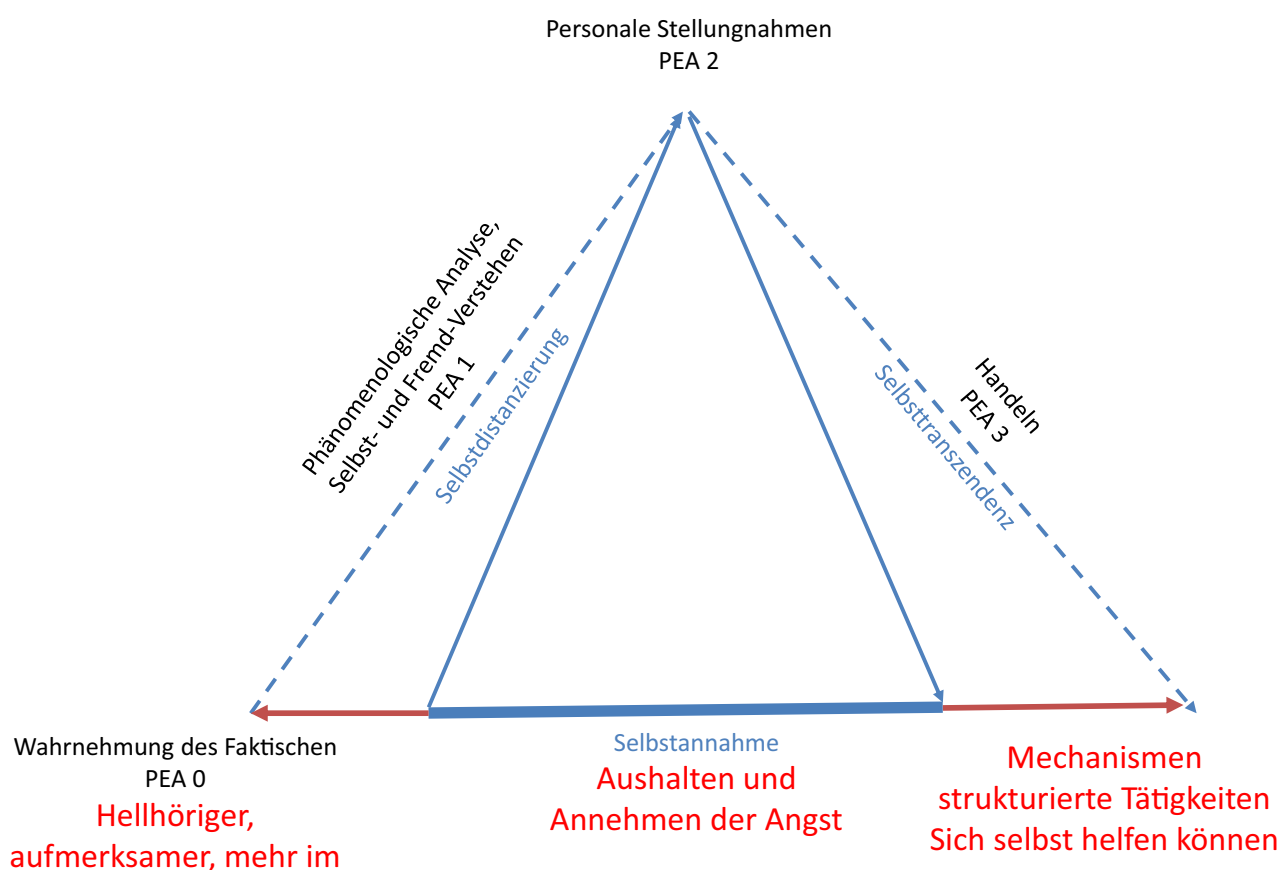

Hier und Jetzt
Die Therapie setzte dementsprechend beim Fokussieren der Wahrnehmung an (siehe Abb. 6) - auf Basis einer annehmenden, stützenden therapeutischen Beziehung, wodurch die Patientin erfuhr, dass sie die Angst aushalten konnte - und beim übenden Handeln sowohl in der Imagination als auch im Alltag. Das Ergebnis kann man sich als Verbreiterung der ontologischen Basis vorstellen, die eine breitere Abstützung des Ich gegenüber den Ängsten bildet.

Eine weitere Passage erschließt sich vor allem aus der phänomenologischen Betrachtung. Dazu sollen vorerst weitere Informationen zu Elisabeths Lebenssituation gegeben werden: Elisabeth ist Ziviltechnikerin wie ihr Mann und half in den letzten Jahren in der Firma mit. Elisabeth über die Entstehung ihrer Ängste:

E: „Die Einsamkeit, also, ja, wenn ich schon Jahre (lacht) zuhause sitze. ... Weil es mir vor Augen hält, ich habe mich nicht weiterentwickelt, ich bin eine Hausfrau, o.k. ich bin für mein Kind da, das wollte ich ja. ... ich bin halt in der Entwicklung stehen geblieben. ... das hat mich fertiggemacht ... Oh mein Gott. Jahre, jetzt so viele Jahre zu Hause."

Hier wird eine tiefe Erschütterung spürbar (vor allem aus den Worten „Oh mein Gott“) - eine Erschütterung, zu wenig gelebt zu haben. Das Lachen passt auf den ersten Blick nicht zur Erschütterung. Es ist ein Lachen, das die Wucht der Erkenntnis etwas abzumildern und tragbar zu machen vermag.

Dann folgt wieder eine kognitive Erklärung zur Entstehung der Ängste. In der Betonung der Worte „gar nichts“ liegt ein erstes, zaghaftes emotionales SichAufbäumen:
„[In der Familie ist] mir ja eigentlich nicht zu viel zugetraut worden ... da habe ich eigentlich nie selber kämpfen müssen ... Und die Ängste sind auch daraus entstanden, dass die Mutter, die einem immer das eingeredet hat, ihre Angst vor [Krankheiten]. Sie hat ja Krebs gehabt und hat ja nie darüber geredet. Und daraus [sind] auch meine Ängste vor Krankheiten entstanden, weil ja in der Familie nichts besprochen wurde. Gar nichts. "

In der Erschütterung und im Sich-Aufbäumen kommt sich Elisabeth näher, sie lässt sich auf ihre Gefühle ein und kommt damit zu Stellungnahmen, die in der Emotion gegründet sind und damit robuster gegen Anflutungen von Ängsten.

Eine weitere wichtige Veränderung ist Elisabeths gelassenerer Umgang mit ihrer chronischen Erkrankung Multiple Sklerose - hier könnte der wesentliche Auslöser für die starke Angstsymptomatik zu Therapiebeginn gelegen sein. Hier wechselt die Fragestellung $\mathrm{zu}$ „Was hat sich verändert?" Die wichtigste Veränderung: Die Therapie hat Elisabeth ermöglicht, in eine Distanz zur Angst vor der MS zu kommen und andere Ursachen für ihre Symptome zuzulassen.

E: „Noch ein Thema mit der Angst ist diese chronische Erkrankung, die ich habe. ... Weil das ist, immer wie so ein Damoklesschwert und immer wieder, wenn ich jemanden im Rollstuhl sehe, denk ich mir ,oh mein Gott, MS“... Wenn man eine Erkrankung hat, die, wo man nicht weiß, wie der Verlauf immer sein könnte, kann ja alles sein. ... Es waren ja auch die Angst-Symptome, die sind ja genau dorthin gefahren, wo die MS ihre Symptome hinlegt, also in den Beinen, Taub- 
heitsgefühle, Schwindel. ... So hat das eigentlich angefangen.

I: Und wie würden Sie es heute einschätzen? War das eine MS-Symptomatik oder wie sehen Sie das?

E: Vielleicht, sagen wir, war es ein kleiner Schub. Aber ... das mit diesen körperlichen Symptomen, das waren die Ängste. Weil sie genau dort, Angst vor Rollstuhl, also vor Taubheit und die sind da reingefahren. Also, das glaube ich, hat wirklich meine Psyche gemacht. Da bin ich mir sicher. Vielleicht war es ein Hauch, ein Schub, ich bin nicht sicher. Also rückblickend auf die Jahre, wo ich dann und wann einen Schwindel gehabt habe, waren vielleicht auch Ängste. Also damals, wo das Cortison teilweise gar nicht geholfen hat."

Die Veränderung in Bezug auf die MS-Erkrankung ist sicher die stärkste Veränderung im Laufe der Therapie.

Insgesamt könnte man sagen, dass Elisabeth durch die Therapie ein vertieftes Verständnis für sich selbst entwickelt und größeren Abstand zu ihren Ängsten gewonnen hat. Sie ist insgesamt offener in der Wahrnehmung und freier im Handeln geworden.

\section{Zusammenfassung und Ausblick}

Die vorliegende Arbeit veranschaulicht, wie eine phänomenologisch-hermeneutische Diagnostik, die den Psychotherapie-Prozess einleitet, begleitet und rückblickend $\mathrm{zu}$ verstehen versucht, gleichzeitig die Prozess-Outcome-Forschung um substantielle Erkenntnisse bereichern kann. Die Personale Existenzanalyse nach Längle liefert ein aussagekräftiges Modell für den Forschungsprozess, für das therapeutische Vorgehen sowie für das Diagnostizieren als Schnittstelle zwischen beiden.

Eine phänomenologisch-hermeneutische Analyse liefert einen Zugewinn an klinischem Wissen für die Praktikerin. Dementsprechend sollte die Zukunft der Psychotherapie-Forschung in einer stärkeren Verbindung von klinischer Praxis und Forschung liegen.

Interessenkonflikt A. Görtz gibt an, dass kein Interessenkonflikt besteht.

Open Access Dieser Artikel wird unter der Creative Commons Namensnennung 4.0 International Lizenz veröffentlicht, welche die Nutzung, Vervielfältigung, Bearbeitung, Verbreitung und Wiedergabe in jeglichem Medium und Format erlaubt, sofern Sie den/die ursprünglichen Autor(en) und die Quelle ordnungsgemäß nennen, einen Link zur Creative Commons Lizenz beifügen und angeben, ob Änderungen vorgenommen wurden.

Die in diesem Artikel enthaltenen Bilder und sonstiges Drittmaterial unterliegen ebenfalls der genannten Creative Commons Lizenz, sofern sich aus der Abbildungslegende nichts anderes ergibt. Sofern das betreffende Material nicht unter der genannten Creative Commons Lizenz steht und die betreffende Handlung nicht nach gesetzlichen Vorschriften erlaubt ist, ist für die oben aufgeführten Weiterverwendungen des Materials die Einwilligung des jeweiligen Rechteinhabers einzuholen.

Weitere Details zur Lizenz entnehmen Sie bitte der Lizenzinformation auf http://creativecommons.org/licenses/by/4. $0 /$ deed.de.

\section{Literatur}

Braakmann, D. (2015). Historical Paths in Psychotherapy Research. In O.C.G. Gelo, A. Pritz \& A. Rieken (Hrsg.), Psychotherapy Research (S.39-65). Wien:Springer.

Eco, U. (1993). Wie man eine wissenschaftliche Arbeit schreibt. Doktor-, Diplom- und Magisterarbeit in den Geistes- und Sozialwissenschaften. Heidelberg: UTB.

Elliott, R. (1999). Client change interview protocol. http:// www.experiential-researchers.org/instruments/elliott/ changei.html/.Zugegriffen: 18.Apr. 2020.

Fischer, G. (2011). Psychotherapiewissenschaft. Einführung in eine neue humanwissenschaftliche Disziplin. Gießen: Psychosozial Verlag.

Franke, G. H. (2014). Symptom-Checklist-90-Standard (SCL90-S). Göttingen: Hogrefe.

Görtz, A. (2019). „Was hat mir geholfen?“ - Wirkelemente im Therapieprozess aus Patientensicht. Ein Beitrag zur Psychotherapie-Prozessforschung. Existenzanalyse, 36(1), 15-43.

Längle, A. (1999). Die anthropologische Position der Personalen Existenzanalyse (PEA). Existenzanalyse, 16/1, 18-25.

Längle, A. (2005). Handhabung und Verwendung der Diagnostik aus Sicht der Existenzanalyse. In H. Bartuska, M. Buchsbaumer, G. Mehta, G. Pawlowsky \& S. Wiesnagrotzky (Hrsg.), Psychotherapeutische Diagnostik. Leitlinien für den neuen Standard (S. 85-92). Wien: Springer.

Längle,A. (2013). Lehrbuch zur Existenzanalyse - Grundlagen. Wien: Facultas.

Längle, A., Orgler, Ch , \& Kundi, M. (2000). Die Existenzskala. Göttingen: Hogrefe.

Hinweis des Verlags Der Verlag bleibt in Hinblick auf geografische Zuordnungen und Gebietsbezeichnungen in veröffentlichten Karten und Institutsadressen neutral. 